1996 IEEE International Conference on Multisensor Fusion and Integration for Intelligent Systems, Washington D.C., December 8-11, 1996.

\title{
Knowledge Assistant: A Sensor Fusion Framework for Robotic Environmental Characterization RECEIVED
}

\author{
Sandia National Laboratories \\ Albuquerque, NM 87185
}

John T. Feddema, J. Jill Rivera, and Susan D. Tucker ${ }^{*}$

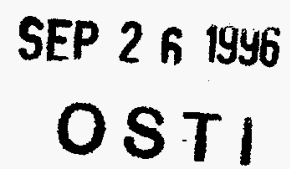

\begin{abstract}
A prototype sensor fusion framework called the "Knowledge Assistant" has been developed and tested on a gantry robot at Sandia National Laboratories. This Knowledge Assistant guides the robot operator during the planning, execution, and post analysis stages of the characterization process. During the planning stage, the Knowledge Assistant suggests robot paths and speeds based on knowledge of sensors available and their physical characteristics. During execution, the Knowledge Assistant coordinates the collection of data through a data acquisition "specialist." During execution and post analysis, the Knowledge Assistant sends raw data to other "specialists," which include statistical pattern recognition software, a neural network, and modelbased search software. After the specialists return their results, the Knowledge Assistant consolidates the information and returns a report to the robot control system where the sensed objects and their attributes (e.g. estimated dimensions, weight, material composition, etc.) are displayed in the world model. This paper highlights the major components of this system.
\end{abstract}

\section{Introduction}

Over the past five years Sandia National Laboratories (SNL) has been developing robotically deployed site characterization systems for DOE facilities such as Hanford and INEL [1,2]. While these systems are good at gathering and storing large volumes of sensor data, final analysis of the data still requires a team of chemists and physicists. The objective of this current project is to develop a framework and a set of general tools which will simplify and speed up sensor data analysis and warn the operator of impending dangers or malfunctions. The software and algorithms developed should reduce the time and expense of the characterization process by minimizing the number of on-site technical personnel required to collect and interpret large volumes of complex, multivariate sensor data. This generic sensor fusion framework is to be applied across a wide range of characterization activities including buried waste, underground storage tanks, laboratory analysis, and decommissioned facilities.

A sensor fusion (or data interpretation) framework could play a vital role in automating the characterization process. Figure 1 illustrates the robotic site characterization life cycle. The data interpretation module starts with knowledge of regulatory requirements, knowledge of the available sensors, and an estimate of the site contents. From this information, an expert system formulates a risk assessment and data acquisition plan. These plans are reviewed and modified by the site manager. The data acquisition plan includes sampling rates of the equipment and spacing and patterns of scans which are directly translated into robot paths and instrumentation control sequences. While the robot is performing the motion and the data acquisition system is collecting the data, the data interpretation module looks at the data using various statistical, model-based, and learning tools. A new estimate of the site contents is continuously generated and rules on regulatory requirements are used to modify the risk assessment and data acquisition plan. As risk increases, the operator is warned; and if risk reaches a certain threshold, the operation is automatically stopped.

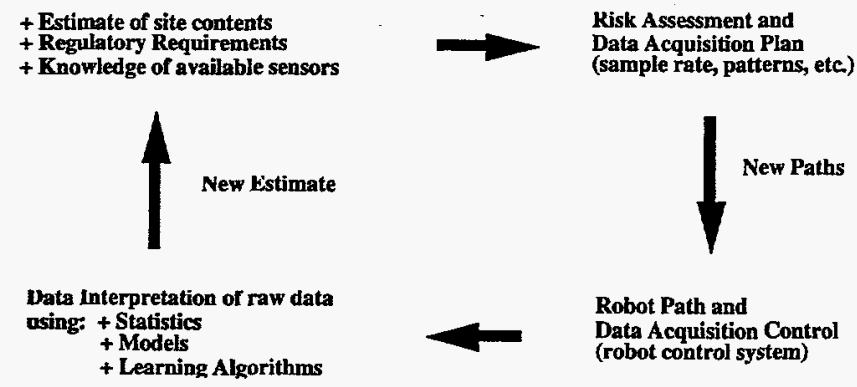

Figure 1. Robotic site characterization life cycle.

\footnotetext{
* This work performed at Sandia National Laboratories supported by the U.S. Department of Energy under contract DE-AC0494AL85000.
} 


\section{DISCLAMMER}

Portions of this document may be illegible in electronic image products. Images are produced from the best available original document. 


\section{DISCLAIMER}

This report was prepared as an account of work sponsored by an agency of the United States Government. Neither the United States Government nor any agency thereof, nor any of their employees, makes any warranty, express or implied, or assumes any legal liability or responsibility for the accuracy, completeness, or usefulness of any information, apparatus, product, or process disclosed, or represents that its use would not infringe privately owned rights. Reference herein to any specific commercial product, process, or service by trade name, trademark, manufacturer, or otherwise does not necessarily constitute or imply its endorsement, recommendation, or favoring by the United States Government or any agency thereof. The views and opinions of authors expressed herein do not necessarily state or reflect those of the United States Government or any agency thereof. 
When investigating how a sensor fusion framework might be structured, we first analyzed the various stages of a data interpretation process. After discussing this issue with several experts in the field, the following general stages of data interpretation became apparent.

1. Low level data manipulation. For example, interpolators, data croppers, filtering algorithms, and transformations.

2. Extraction of features from the data either using signal processing techniques or physical sensor models. For example, the peaks and areas of spectral data, such as Gas Chromatography (GC), Raman, infra red, and gamma spectroscopy, are used to characterize data. Another example is the extraction of features within an aerial image to locate landmarks on a buried waste site.

3. Classification of data using techniques such as Bayesian hypothesis testing, DempsterShafer, Fuzzy Logic, and Neural.Networks.

4. Heuristic expert system rules to guide the previous levels, make high level control decisions, provide operator guidance, and provide early warnings and diagnostics.

A pictorial example of the information flow within a data interpretation framework is shown in Figure 2. Many of the early stages are sensor specific. However, if formulated correctly; many of the higher level stages are reusable across many different sensing modalities. For example, many of the spectral analysis tools are common for GC, gamma, or Raman data interpretation. Also, the same classification tools can be used for classifying GC spectral as can be used for identifying barrels from GPR (Ground Penetrating Radar) data.

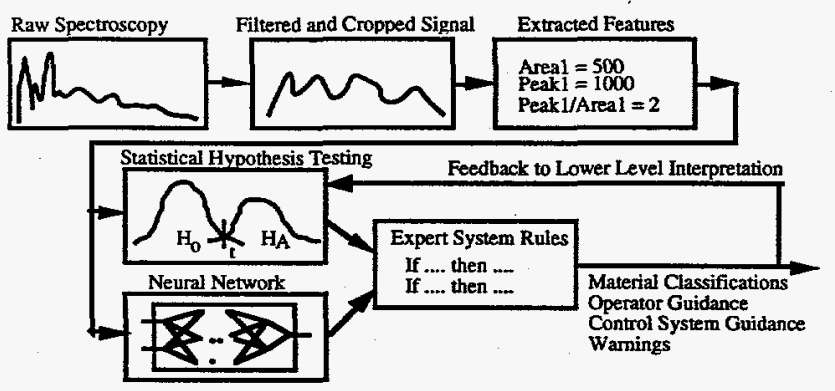

Figure 2. Data Interpretation with Statistical, Learning, and Expert System Reasoning.

Based on this analysis, we began development on a "Knowledge Assistant" sensor fusion framework which contains an expert system at the top level, and various specialists at the lower levels (see Figure 3). The Knowledge Assistant helps the operator of a robotic characterization system, much like a co-pilot helps a pilot navigate an airplane. The Knowledge Assistant performs the job of the chemist and physicist by determining how to scan over the environment based on the sensor platform available and the regulatory requirements. The Knowledge Assistant also assists in the collection and post analysis of the data. Aiding the Knowledge Assistant are various specialists which can be reconfigured based on the application. When making decisions, the Knowledge Assistant directs information to the lower levels and provides the final fusion of the results from the specialists. As an initial test, we developed four specialists:

- Data Acquisition Specialist

- Statistical Pattern Recognition Specialist

- Neural Network Classifier Specialist

- Gradient-Based Model Fitting Specialist Each specialist runs on a separate computer and communicates with the Knowledge Assistant via a client-server connection. This last specialist is New Mexico State University's Multi-sensor Analysis Program for Environmental Restoration (MAPER) system, and more detailed information can be found in [3].

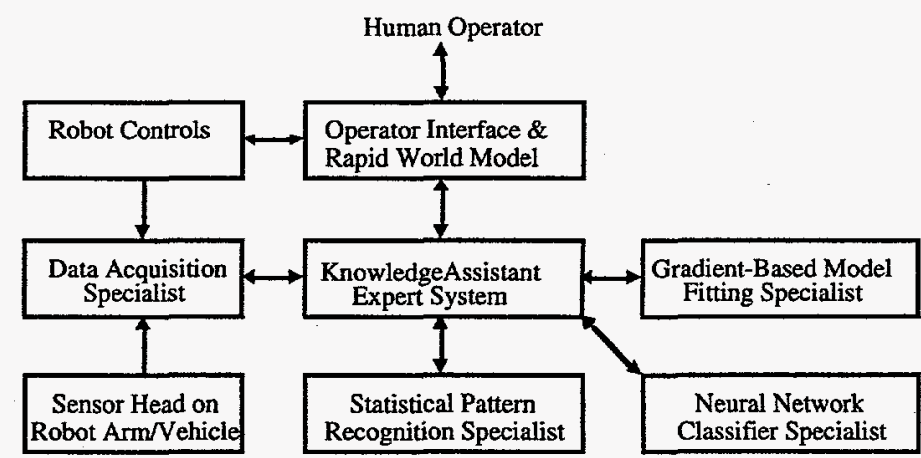

Figure 3. Knowledge Assistant Architecture.

The next three sections discuss the Knowledge Assistant, the Statistical Pattern Recognition Specialist, and the Neural Network Classifier Specialist. Conclusions and suggestions for future work are described in the final section.

\section{Knowledge Assistant}

The Knowledge Assistant was developed on a realtime expert system [4]. Through the expert system, heuristic reasoning in the form of "if-then" rules can be added to the system's knowledge base. The Knowledge Assistant also directs the flow of data to the appropriate analysis routines. For example, when analyzing magnetometer data, the expert system sends the data to a gradient search algorithm which fits the raw sensor data to a model. Expert system rules are also used to set the initial boundary conditions and residual thresholds of the search. 
When categorizing soils, the expert system directs data to a statistical pattern recognition routine which characterizes soils based on tip force, pore pressure, viscosity, and $\mathrm{pH}$ sensor readings from a cone penetrometer.

To test the capabilities of the Knowledge Assistant, we presented the system with the task of robotically scanning over a mock buried waste site with a sensor package containing a magnetometer, pyrometer, and camera. The objective was to map out the environment, display it on a graphical screen, and list attributes (such as temperature and metallic content) with each detected target. The sequence of events which occurred between the Knowledge Assistant and its specialists is described below.

The Knowledge Assistant first receives the bounding box information (the volume that the operator would like to scan) from the world model. User supplied information such as the scan height and resolution information of each sensor are stored in the knowledge base of the Knowledge Assistant. Several rules in the knowledge base combine these sensor requirements to determine the robot path and speed. This information is passed back to the world model where the robot motion is graphically previewed by the operator. If accepted by the operator, the robot begins its path.

The raw sensor data along with the position and orientation of the robot is collected in files by the data acquisition specialist. The data acquisition specialist can also send real-time data to other specialists, such as the statistical pattern recognition specialist, for real-time classification. Live data can be displayed on strip charts and regional maps by the data acquisition specialist if desired. When a robot pass is completed, the Knowledge Assistant sends the file information to the other specialists for analysis of the sensor data. Magnetometer and pyrometer data files are sent to the MAPER specialist as soon as the Knowledge Assistant receives the data files names. The Knowledge Assistant receives statistical information from MAPER for each of the files, executes an additional set of rules and sends threshold data back to MAPER to help guide its analysis. When the MAPER analysis is completed, target file names are received from MAPER. The Knowledge Assistant reads a target file in as an instance. The process is repeated as each robot pass is completed.

When three robot passes have been completed and the files names have been received, the Knowledge Assistant sends the files names to the neural net specialist. The neural net specialist will analyze the data and return the target file to the Knowledge
Assistant . The Knowledge Assistant reads the target file in as an instance.

After all target files are recieved from the MAPER, neural network, and statistical pattern recognition specialists, the Knowledge Assistant consolidates the results. A target is defined by $x 1, y 1, z 1$, radius $1, x 2$, $\mathrm{y} 2, \mathrm{z} 2$, radius 2 , magnetometer value, and pyrometer value. The target may not have radius values or only one sensor magnitude value. The Knowledge Assistant rules check each target instance attribute created by one file with each target instance attribute from the first target file. If any bounding boxes overlap or touch, the targets are combined into one target with an encompassing bounding box and with attribute values which are the weighted sum of the individual targets. If the bounding boxes do not overlap, the target attributes are added to the first target file. This process continues until all of the target files have been checked against the first target file. Reports are generated to convey the number of targets before and after consolidation. A file is then created by the Knowledge Assistant which contains the consolidated target data. The file name is sent to the world model where the target objects and their attributes are displayed in a three-dimensional graphic environment.

\section{Statistical Pattern Recognition Specialist}

This section briefly describes how statistical pattern recognition techniques were used to classify the objects that the magnetometer and pyrometer sensed. The objective was to classify the materials in realtime as metal, hot non-metal, hot metal, and no target. These same pattern recognition techniques have also been used to classify simulated waste from an Underground Storage Tank using a cone penetrometer. In that case, the measurements of sensors such as sleeve friction stress, tip stress, friction ratio, and pore pressure were used to discriminate between air, supernate, sludge, and salt cake.

The statistical pattern recognition software was constructed to be used in two phases. During a learning phase, either simulated sensor readings or actual measurements from live sensors are used to learn the mean and variance of the sensor data in a particular class (e.g. metal, hot non-metal, hot metal, and no target). During a follow-on monitoring phase, a multi-variate, multi-class Bayesian classifier is used to identify the class. The results of the classifier are used by the expert system to notify the remote operator of the type of material sensed and any suggested plan of action. The minus-loglikelihood value indicates to the operator the probability of correct classification. 
A statistical pattern recognition technique, known as Bayesian hypothesis testing [5], is used to analyze sensor measurements and discriminate between classes. This method of classification is well recognized within the field of safeguards and nuclear material management $[5,6,7]$. Below is a brief introduction to the subject. A more detailed discussion can be found in [8].

Bayesian hypothesis testing is used to classify a sample $X$ of dimension $n$ (i.e., $X \in \Re^{n}$ ) into one of $m$ classes $\omega_{i}, i=1, \ldots, m$. The samples are assumed to come from a Gaussian (Normal) distribution with a mean and covariance which are learned prior to on-line testing. The decision rule for multiple classes is as follows: A sample $X$ is an element of class $\omega_{i}$ if

$$
P\left(\omega_{i} \mid X\right)>P\left(\omega_{j} \mid X\right) \quad \forall j=1, \ldots, m ; j \neq i .
$$

where $P\left(\omega_{i} \mid X\right)$ is the conditional probability that class $\omega_{i}$ occurred given sample $X$. The above equation selects the class which has the greatest probability.

Using Bayes' theorem, the conditional probability $P\left(\omega_{i} \mid X\right)$ can be replaced with $P\left(\omega_{i}\right) p\left(X \mid \omega_{i}\right)$ where $P\left(\omega_{i}\right)$ is the a priori probability that class $\omega_{i}$ will occur, and $p\left(X \mid \omega_{i}\right)$ is the $a$ priori conditional density function that sample $X$ will occur if we know the class of $X$ is $\omega_{i}$.

Assuming Gaussian distributions of $X$, the decision rule can be rewritten as: A sample $X$ is an element of class $\omega_{i}$ if

$$
h_{i j}(X)<\ln \left[\frac{P\left(\omega_{i}\right)}{P\left(\omega_{j}\right)}\right] \quad \forall j=1, \ldots, m ; j \neq i .
$$

The minus-log-likelihood is given by

$$
\begin{aligned}
& h_{i j}(X)=0.5\left(X-M_{i}\right)^{t} C_{i}^{-1}\left(X-M_{i}\right) \\
& -0.5\left(X-M_{j}\right)^{t} C_{j}^{-1}\left(X-M_{j}\right)+0.5 \ln \left[\frac{\operatorname{det}\left(C_{i}\right)}{\operatorname{det}\left(C_{j}\right)}\right]
\end{aligned}
$$

where $M_{i}$ is the mean of $X$ given class $\omega_{i}$ and $C_{i}$ is the covariance matrix of $X$ given class $\omega_{i}$. The superscripts $t$ and -1 denote matrix transpose and inverse operations. The function $\operatorname{det}()$ denotes the determinant of the matrix.

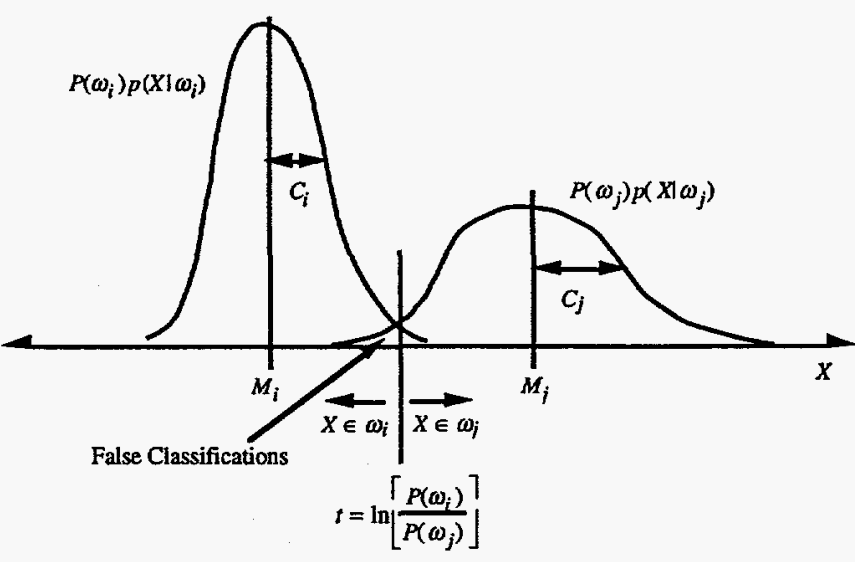

Figure 4. Uni-variate, two-class example of Bayesian hypothesis testing.

Figure 4 illustrates a uni-variate (in $X$ ), two-class example of Bayesian hypothesis testing. The area under each curve is equal to the $a$ priori probability of class $\omega_{i}$. The sum of these areas for all classes is one. The value $t$ is the threshold below which we assign the sample to class $\omega_{i}$, and above which we assign the sample to class $\omega_{j}$. The area of overlap of the two curves is the probability of false classification. To achieve the best classification, it is desirable to minimize the probability of false classification. This usually means that you would like the means of the two classes to be far apart and the covariances to be small (i.e. small standard deviation). Since the probability of error is computed during the learning phase (when the means and covariances are learned), it may be used as a measure for choosing the sensors which provide the best classification.

The total probability of error between classes $\omega_{i}$ and $\omega_{j}$ can be estimated by

$$
\varepsilon_{i j_{\text {total }}}=P\left(\omega_{i}\right) \varepsilon_{1 i j}+P\left(\omega_{j}\right) \varepsilon_{2 i j}
$$

where the errors $\varepsilon_{1 i j}$ and $\varepsilon_{2 i j}$ are associated with incorrectly choosing $\omega_{i}$ when we should have chosen $\omega_{j}$ and vice versa [8]. Note that the above equation is only an estimate of the probability of error since the minus-log-likelihood is not Normal but instead quadratic when $C_{i} \neq C_{j}$. Also, note that this equation provides a pair wise comparison between classes. These values can be put in matrix form as shown below. 


$$
\left[\begin{array}{cccc}
0 & \varepsilon_{12_{\text {total }}} & \varepsilon_{13_{\text {total }}} & \varepsilon_{14_{\text {total }}} \\
\varepsilon_{12_{\text {total }}} & 0 & \varepsilon_{23_{\text {total }}} & \varepsilon_{24_{\text {total }}} \\
\varepsilon_{13_{\text {total }}} & \varepsilon_{23_{\text {total }}} & 0 & \varepsilon_{34_{\text {total }}} \\
\varepsilon_{14_{\text {total }}} & \varepsilon_{24_{\text {total }}} & \varepsilon_{34_{\text {total }}} & 0
\end{array}\right]
$$

By looking across a row, we can see which classes appear to overlap causing larger error terms. By adding up $i$ th row, we can estimate the total error associated with choosing class $\omega_{i}$.

Overall, we found that the statistical pattern recognition technique worked well if the sensor readings were repeatable and stable. For example, we had a very high detection rate using the magnetometer and pyrometer. Unfortunately, when measuring the simulated Underground Storage Tank waste, the penetrometer did not always return repeatable results.- The viscosity measurement was different depending on the sequence of events (e.g. inserted in sludge and then salt cake, or first inserted in salt cake and then sludge). Also, since we taught the system based on the steady state sensor readings, we were not able to capture transient responses such as ringing which occurred when the penetrometer entered the salt cake. These two results imply that in the future we should add some time dependent features (e.g. tip force readings at the current time and some delayed time) to the sample $X$. Another solution is that the expert system could detect when the sensor readings were not stable and could switch to different learned parameters based on the situation at hand.

Another problem with Bayesian classification is that there is no way to capture a sense of non belief. In other words, we should be able to say that I do not believe that it is any of the four classes, and therefore, it must be something else. In the future, we may be able to solve this by using DempsterShafer belief functions.

\section{Neural Network Specialist}

In our tests, the goal of the neural network specialist was to map heat sources in an area by examining pyrometer data from multiple scans over the area. The pyrometer provides a measurement of the average temperature within its field of view. In our case, we used a pyrometer with a 1:2 field of view. This means that the spot diameter is one-half the distance between the pyrometer and the waste surface. Because of this averaging effect, the temperature map created by scanning the pyrometer over the surface becomes blurred as the distance between the pyrometer and the waste increases. The neural network takes the raw sensor maps at three heights and determines the number, location, size, height, and temperature of sources. This work is also applicable to other radiation sources, given suitable sensors.

The reason that a neural network was used for this task was to evaluate how learning algorithms could be used to analyze sensor readings over time. The hypothesis is that expert knowledge will be required to analyze the first few waste sites. However, learning algorithms will be useful as more information becomes available over time. Learning sets from previous sites will be used to train the neural networks. Eventually, the neural networks will be able to perform a higher quality analysis of extremely complex, non-linear, multi-variate data.

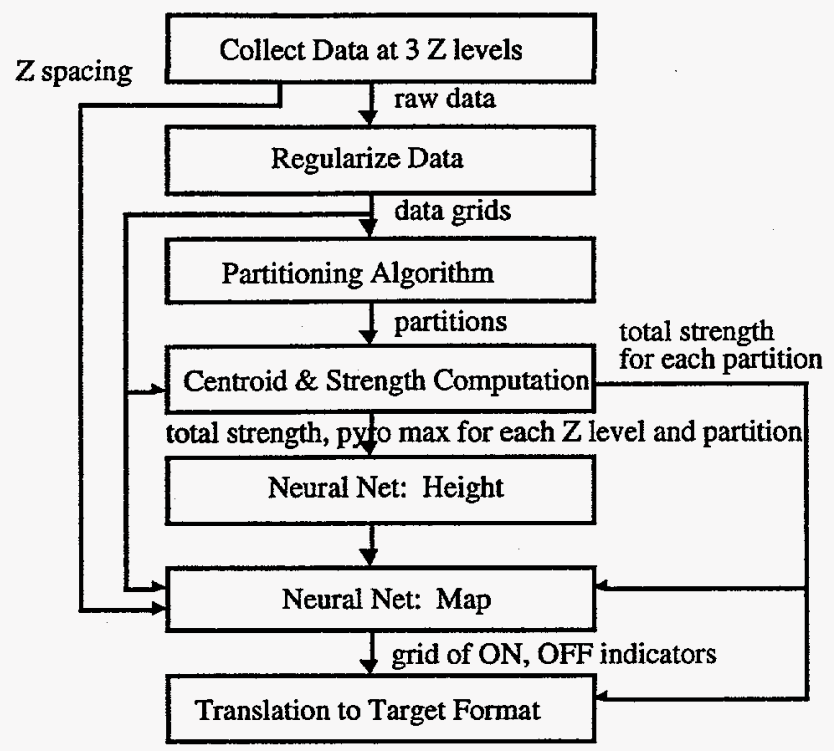

Figure 5. Flow diagram for neural network processing of pyrometer data

Figure 5 is a flow chart of the neural network training process in our tests. During data collection, the sensing axis of the pyrometer is pointed straight down and is moved by the robot arm in a serpentine scan pattern over the selected area. The algorithms developed here require scans regularly spaced in $\mathrm{Z}$ (height). Temperature data is collected at regular time-sampling intervals; however, since the speed of the robot changes, the data is not collected on an evenly spaced grid. Therefore, the next step, "Regularize data," creates a regularly spaced grid of pyrometer data for each $\mathrm{Z}$ level. Regularized data for one pyrometer scan is shown in Figure 6. White lines indicate edges of heat sources: a pipe wrapped with heat tape, and two rectangular heat sources at 400 degrees $F$ and 250 degrees $F$. Three scans were taken 6 inches apart in height. The rectangular heat 
sources were 17.7 inches and 18.1 inches beneath the lowest scan plane.

Next, a partitioning algorithm is applied to the lowest level scan. The number of sources are identified, and each square in the grid is assigned to a partition. Two partitions are identified, each encompassing one of the rectangular heat sources. The heated pipe did not generate sufficient signal for it to be picked out by the partitioning algorithm. The centroid and strength computation algorithms are applied to each partition. The strength is defined as the sum of the readings in the partition. Note that this is not the radiant power, which is a function of absolute temperature to the fourth power.

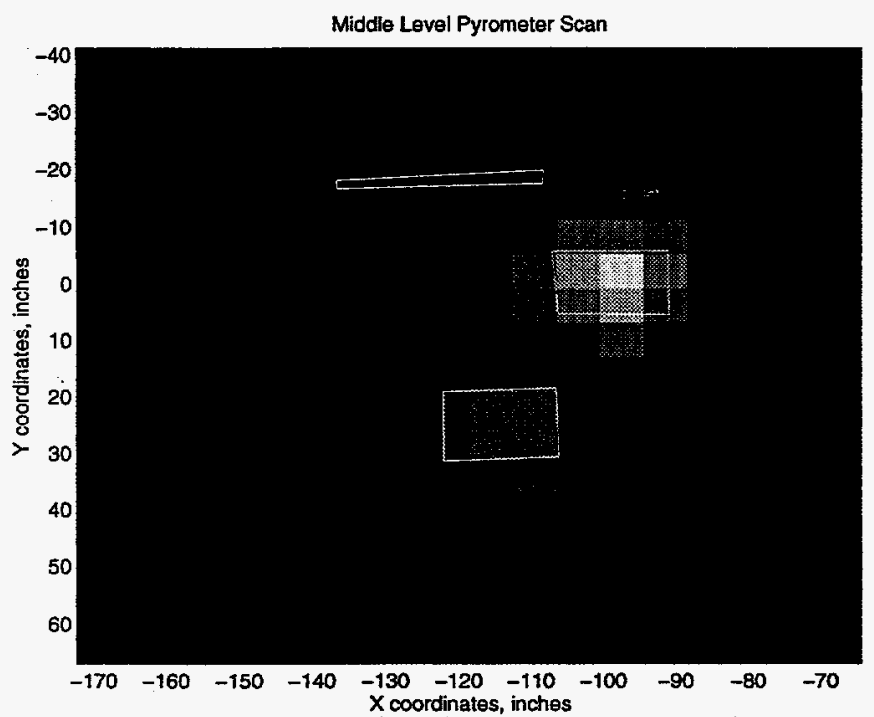

Figure 6. Middle level pyrometer scan data.

The height determination network shown in Figure 7 is run once for each partition. This is a two layer, fully connected, feedforward network that has been trained by a backpropagation algorithm [9]. The sizes of the boxes represent the last value through the network at that node, and whether the box is shaded or empty implies the value is positive or negative. The weights at each node are the result of the backpropagation technique. Inputs to this network are (a) strength estimate for the partition (POW), and (b) peak pyrometer readings for each $\mathrm{Z}$ level for the partition (PK1, PK2, PK3). The network output is an estimate of the distance from the lowest measurement plane to the plane of the source.

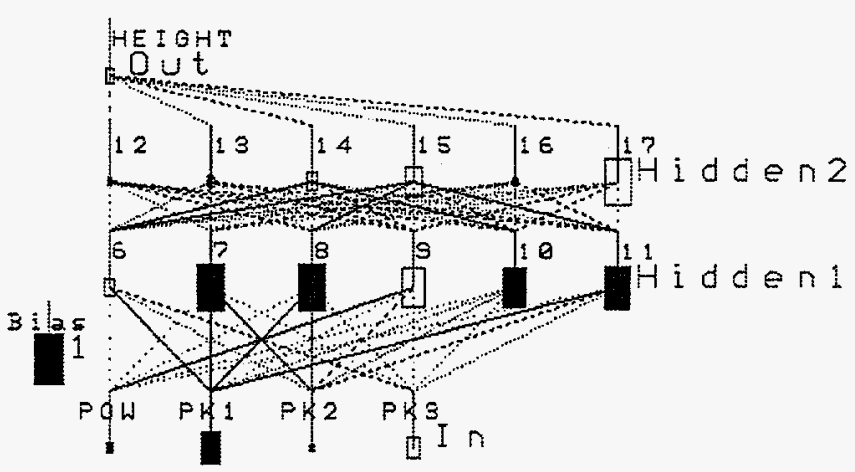

Figure 7. Height determination network.

Similarly, a mapping network is executed once for each cell in the $X-Y$ grid. This is also a two layer, fully connected, feedforward network that has been trained by a backpropagation algorithm. Inputs are (a) the distance between scans in the $\mathrm{Z}$ direction, (b) the height from the height determination network, and (c) the pyrometer readings for this location on the grid for each scan. The network output MAP is interpreted as a binary signal, 1 indicating presence of the source at this cell, and 0 indicating absence. The results on the example problem are shown in Figure 8. White cells indicate presence of a source, black indicates absence.

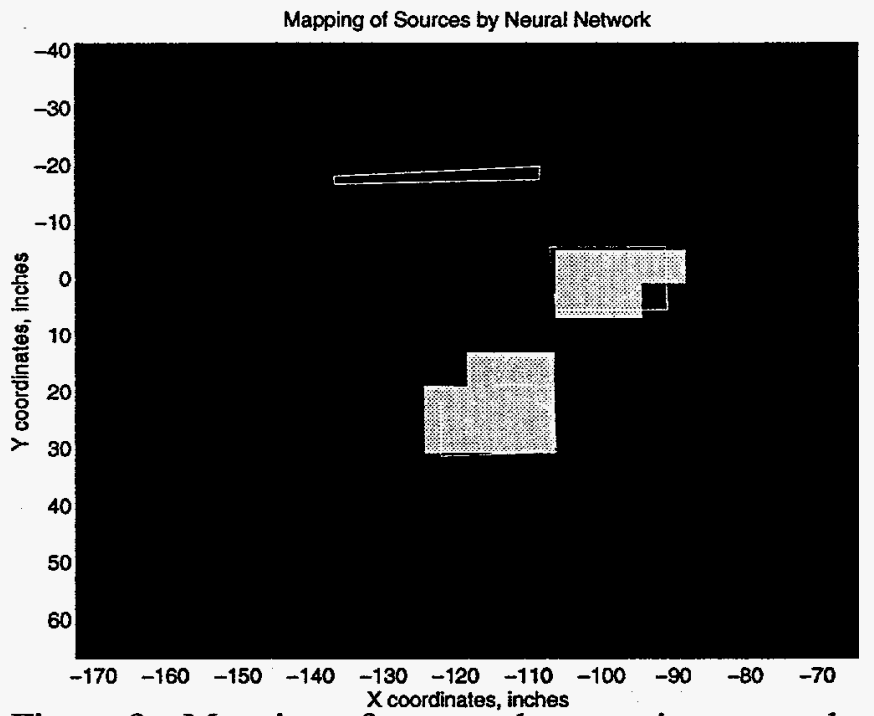

Figure 8. Mapping of sources by mapping network.

The last step of the process is to translate the results into the target file format, indicating the height of each source and its maximum extents in an X-Y coordinate system. The temperature of each source is the power estimate divided by the number of cells indicated by the mapping network (in other words, the average temperature measured per identified object). 
The height and mapping neural networks were trained by dividing 24 experiments into test and training data sets so that generalization could be examined [10]. Performance of the height network is shown in Table 1. Training was stopped when performance on the test set began to decline. The imperfect generalization seen here indicates that more training data would be needed to improve the accuracy of the network on non-training examples.

The output of the mapping network is deemed to be 1 if the center of the cell is within the borders of a source. Hence some errors are to be expected in cells near the edges of a source. Performance of the mapping network is tabulated in Tables 2 and 3. Tabulated figures indicate the number of examples in each category. The training set was selected so that the numbers of 1 and 0 samples were equal, to keep the network from being biased toward zero valued outputs. This left an imbalance of 1 and 0 entries in the test set, as reflected in Table 3.

Table 1. Height network results.

\begin{tabular}{|l|l|l|l|}
\hline & $\begin{array}{l}\text { STD error } \\
\text { (inches) }\end{array}$ & $\begin{array}{l}\text { MIN error } \\
\text { (inches) }\end{array}$ & $\begin{array}{l}\text { MAX } \\
\text { error } \\
\text { (inches) }\end{array}$ \\
\hline Training Set & 1.13 & -2.4 & +1.5 \\
\hline Test set & 2.18 & -9.3 & +3.8 \\
\hline
\end{tabular}

Table 2. Mapping network results on training set.

\begin{tabular}{|l|l|l|}
\hline & $\begin{array}{l}\text { Actual 1 } \\
\text { Output }\end{array}$ & $\begin{array}{l}\text { Actual 0 } \\
\text { outputs }\end{array}$ \\
\hline $\begin{array}{l}\text { Desired 1 } \\
\text { Output }\end{array}$ & 1919 & 127 \\
\hline $\begin{array}{l}\text { Desired 0 } \\
\text { Output }\end{array}$ & 142 & 1931 \\
\hline
\end{tabular}

Table 3. Mapping network results on the test set.

\begin{tabular}{|l|l|l|}
\hline & $\begin{array}{l}\text { Actual 1 } \\
\text { Output }\end{array}$ & $\begin{array}{l}\text { Actual 0 } \\
\text { outputs }\end{array}$ \\
\hline $\begin{array}{l}\text { Desired 1 } \\
\text { Output }\end{array}$ & 199 & 17 \\
\hline $\begin{array}{l}\text { Desired 0 } \\
\text { Output }\end{array}$ & 103 & 4943 \\
\hline
\end{tabular}

It is easy to see that the approach to mapping here is limited compared to a true deconvolution algorithm. There are several dependencies (such as the height computation depending on the output of the power estimate) that contribute to a cumulative buildup of error as data progresses through the flowchart. Furthermore, the mapping step of this approach ignores the fact that each reading depends not only on the contribution of the source directly beneath the sensor, but also on the contribution of everything within the sensors' field of view.

It seems reasonable that a neural network deconvolution algorithm could be written to better handle these difficulties. That algorithm would consist of a recurrent algorithm that took as inputs both sensor readings and previous outputs of the cell and its neighbors.

\section{Conclusions and Future Directions}

The prototype system described above is an example of the type of automated processing that is desirable for future work in the environmental arena. The combination of automated processing together with intelligent analysis algorithms and a friendly graphical user interface provide a valuable means of improving and speeding up environmental analyses. The expert system provides a high level means to control processing, with potential for future incorporation of additional sensors, further processing, and additional inferencing to determine more accurate results.

We believe that the combination of expert system technology along with statistical hypothesis testing and neural networks provides a valuable means of fusing sensor data. The Bayesian hypothesis testing provides an analytical means of classifying complex multi-variate data, which would otherwise be near impossible to analyze by hand. It can also be used to rapidly select and test different sensor combinations for different characterization tasks. A neural network can be used to accurately model nonlinear, complex relationships. The expert system provides a high level user interface, which can be used to alert operators and inform them of the appropriate next steps.

Future work should focus on the area of data fusion and intelligent inferencing methodologies. This involves incorporating multiple raw data types into a single analysis in order to make better inferences and determine more information than is possible with any single raw data type alone. Goals for this processing include: more accurate location solutions, determination of target material type, identification of target (i.e., 55 gallon steel drum), determination of container status (leaking, intact), and container contents (or lack thereof), etc. This will allow future clean-up operations to be performed more safely, more efficiently, and with better results. 


\section{Acknowledgment}

The authors would like to thank Charles Little and Chris Wilson for providing the rapid world modeling system, visualization tools, and the MAPER interface; Prof. Ed Hensel and his students Jennifer Macy and Brandon Donahue for their help with MAPER; and Dave Fugelso for providing valuable communications software between modules.

\section{References}

[1] Feddema, J.T., "A Miniaturized Sensor System for In Situ Robotic Characterization of Hazardous Waste," SPECTRUM '92, Boise, Idaho, August 23-27, 1992, pp. 75-80.

[2] Feddema, J.T., and Spletzer, B.L., "Generic Data Acquisition System for Robotic Waste Characterization," Proceedings of Space 94, Albuquerque, NM, February 26 to March 3, 1994.

[3] Hensel, E., et. al., "Subsurface Characterization Using Geophysical Methods," International Meeting on Nuclear nad Hazardous Waste Management: SPECTRUM 94, August 1994.

[4] Gensym Corporation, G2 Reference Manual, Version 3.0, 125 Cambridge Park Drive, MA 02140, (617)547-2500.

[5] Avenhaus, Rudolf, Safeguards Systems Analysis, Plenum Press, New York and London, 1986.

[6] Bowen, W. Michael, and Bennett, Carl A., Statistical Methods for Nuclear Material Management, NUREG/CR-4604, The Superintendent of Documents, U.S. Government Printing Office, P.O. Box 37082 , Washington, DC 20013-7082.

[7] Argentesi, F., Avenhaus, R., Franklin, M., and Shipley, J.P., Mathematical and Statistical Methods in Nuclear Safeguards, Hardwood Academic Publishers, New York, 1983.

[8] Fukunaga, Keinosuke, Introduction to Statistical Pattern Recognition, Academic Press, New York and London, 1972.

[9] NeuralWare, Inc, NeuralWorks Professional II/Plus and NeuralWorks Explorer Reference Guide, Version 5.0, Penn Center West, Building IV, Pittsburgh, PA 15276, (412) 787-8222.

[10] Feddema, J.T., Rivera, J.J., Tucker, S.D., and Matek, J.E., "Knowledge Assistant for Robotic Environmental Characterization," SAND961995, August 1996. 